\title{
A theory for the streamwise turbulent fluctuations in high Reynolds number pipe flow
}

\author{
Marcus Hultmark $\dagger$ \\ Department of Mechanical and Aerospace Engineering, Princeton University, Princeton, NJ 08544, USA \\ School of Architecture, Civil and Environmental Engineering, École Polytechnique Fédérale de Lausanne \\ (EPFL), 1015 Lausanne, Switzerland
}

(Received 16 May 2012; revised 15 June 2012; accepted 15 June 2012; first published online 24 July 2012)

A new theory for the streamwise turbulent fluctuations in fully developed pipe flow is proposed. The theory extends the similarities between the mean flow and the streamwise turbulence fluctuations, as observed in experimental high Reynolds number data, to also include the theoretical derivation. Connecting the derivation of the fluctuations to that of the mean velocity at finite Reynolds number as introduced by Wosnik, Castillo \& George (J. Fluid Mech., vol. 421, 2000, pp. 115-145) can explain the logarithmic behaviour as well as the coefficient of the logarithm. The slope of the logarithm, for the fluctuations, depends on the increase of the fluctuations with Reynolds number, which is shown to agree very well with the experimental data. A mesolayer, similar to that introduced by Wosnik et al., exists for the fluctuations for $300>y^{+}>800$, which coincides with the mesolayer for the mean velocities. In the mesolayer, the flow is still affected by viscosity, which shows up as a decrease in the fluctuations.

Key words: Shear layer turbulence, turbulent flows, turbulence theory

\section{Introduction}

Even though it has been almost a century since the scaling laws for turbulent pipe flow were first developed (Stanton \& Pannell 1914), they have received much attention in the last couple of decades. In the late 1980s, questions about the underlying physics were raised, which highlighted the need for better experimental data (see for example George 1988; Frisch \& Orszag 1990; Smits 1991). This motivated improved high Reynolds number test facilities and more accurate measurement techniques. In 1996 the Princeton/ONR Superpipe was first deployed, and the results raised even more questions about the traditional scaling laws. The Superpipe is a unique pipe flow facility that uses pressurized air (up to $220 \mathrm{~atm}$ ) as the working fluid, allowing studies of Reynolds numbers two orders of magnitude higher than previously possible (for more information regarding this facility, see Zagarola 1996; Zagarola \& Smits 1997). The first studies in this facility focused entirely on mean flow measurements, and the results stirred controversy since the mean flow appeared to behave differently from what was expected and what had been observed in previous boundary layer measurements (Zagarola 1996; Zagarola \& Smits 1997; McKeon et al. 2004). The classical theory for the mean velocity profile (developed by Millikan 1939) is based

$\dagger$ Email address for correspondence: hultmark@princeton.edu 
on the argument that the flow can be divided into two regions, an 'inner region' and an 'outer region'. This implies that there is a region where the inner-scaled velocity, $U^{+}=U / u_{\tau}$, can be expressed as a function of the inner-scaled distance from the wall, $y^{+}=y u_{\tau} / \nu$, alone, and another region where the outer-scaled velocity, $\left(U-U_{c l}\right) / u_{\tau}$, can be expressed as a function of the outer-scaled distance, $\bar{y}=y / R$ alone. Both formulations are Reynolds number invariant:

$$
\frac{U}{u_{\tau}}=f_{i}\left(y^{+}\right)
$$

and

$$
\frac{U-U_{c l}}{u_{\tau}}=f_{o}(\bar{y}),
$$

where $v$ is the kinematic viscosity, $u_{\tau}=\sqrt{\tau_{w} / \rho}$ is the friction velocity, $\tau_{w}$ is the wall shear stress, $\rho$ is the fluid density, $U_{c l}$ is the mean centreline velocity and $R$ is the pipe radius. If there is a common region in space where the inner and outer formulations overlap, one can show, by matching derivatives, that the velocity profile will exhibit a logarithmic behaviour:

$$
U^{+}=\frac{1}{\kappa} \log \left(y^{+}\right)+B,
$$

where the von-Kármán constant $\kappa$ is believed to be a universal constant. $B$ is usually referred to as the additive constant, which in the traditional view is Reynolds number independent. However, Wosnik, Castillo \& George (2000) argued that the classical derivation might be valid in the limit of infinite Reynolds numbers, but for realistic flows one cannot assume that the functions are independent of Reynolds number, $R^{+}=\left(R u_{\tau}\right) / \nu$. By allowing $f_{i}$ and $f_{o}$ to also be functions of $R^{+}$they were able to show, by near-asymptotic analysis, that

$$
\left.\bar{y} \frac{\partial f_{o}}{\partial \bar{y}}\right|_{R^{+}}=\frac{1}{\kappa\left(R^{+}\right)}+\left[\left.\frac{\partial f_{i}\left(y^{+}, R^{+}\right)}{\partial \log R^{+}}\right|_{y^{+}}-\left.\frac{\partial f_{o}\left(\bar{y}, R^{+}\right)}{\partial \log R^{+}}\right|_{\bar{y}}\right] .
$$

They further argued that only for wall positions where the term in square brackets (from here on referred to as the sensitivity function for the mean velocity, $S_{m}$ ) vanishes can one expect a logarithmic behaviour. However, here we note that it is enough for the sensitivity function to be constant over a region in space for the mean velocity profile to show a logarithmic profile (although with a different slope). Wosnik et al. further pointed out that the term in square brackets will disappear as Reynolds number tends to infinity, and we recover the classical log law (for more information regarding finite Reynolds number effects see George \& Castillo 1997; Castillo 1997; Wosnik 2000; George 2007). It is important to note that in this theory both $\kappa$ and $B$ are allowed to be functions of $R^{+}$, and $\kappa$ is related to the friction velocity as

$$
\frac{1}{\kappa}=\frac{\mathrm{d}\left(U_{c l} / u_{\tau}\right)}{\mathrm{d} \log R^{+}} .
$$

Another important result from the work of Wosnik et al. is that it allows a shift of the logarithmic behaviour, which yields a solution in the overlap region as

$$
\frac{U}{u_{\tau}}=\frac{1}{\kappa} \log \left(y^{+}+a^{+}\right)+B_{i}
$$


and

$$
\frac{U-U_{c l}}{u_{\tau}}=\frac{1}{\kappa} \log (\bar{y}+\bar{a})+B_{o} .
$$

A shift in the origin can account for two phenomena: first that there might be an underlaying layer in which viscosity is important in the mean equations (the linear and buffer layers); and as well a layer where the dissipative scales are not fully separated from the energy-containing scales. An offset in the logarithm was first introduced by Squire (1948), who used a mixing-length argument to conclude that an offset is needed to include the effect of the viscous sublayer. Wosnik et al. pointed out that only when $y^{+} \gg a^{+}$is the turbulence truly inertial, as recognized in the classical view. The region where $a^{+}$is still noticeable is related to the mesolayer, which was first introduced by George \& Castillo (1997) for boundary layers, and later by Wosnik et al. (2000) for pipe flow. Wosnik et al. further showed that their results fit extremely well to the Superpipe data, but it should be pointed out that the differences with the classical solution are very small and within experimental error. Both Zagarola \& Smits (1997) and McKeon et al. (2004) noticed that the departure from the logarithmic behaviour (on the side closer to the wall) occurred at a higher $y^{+}$value than previously believed, and that in the region $60<y^{+}<500$ the mean velocity appeared to follow a power law:

$$
\frac{U}{u_{\tau}}=C y^{+\gamma}
$$

However, the logarithmic formulation with an offset (such as (1.6)), will have a very similar shape to that of the power law, and Wosnik et al. showed that it can explain the deviation from the classical logarithmic behaviour observed in the Superpipe data.

In this study we are interested in the behaviour of the turbulence fluctuations, specifically the streamwise component, ${\overline{u^{\prime 2}}}^{+}=\overline{u^{\prime 2}} / u_{\tau}^{2}$. Morrison et al. (2004) and Hultmark, Bailey \& Smits (2010) used conventional hot-wire anemometry to investigate the streamwise turbulent fluctuations. The results by Morrison et al. revealed several interesting features, including the appearance of an outer peak in the profile, but the results are difficult to interpret since they are heavily affected by spatial filtering (Hutchins et al. 2009). In order to account for spatial filtering effects Hultmark et al. (2010) used conventional hot wires with matched nondimensional length, $\ell^{+}=\ell u_{\tau} / v$. By matching $\ell^{+}$the spatial filtering (close to the wall) is matched and it can be isolated from the true Reynolds number effects (Smits $e t$ al. 2011). Unfortunately, spatial filtering limits the measurable Reynolds number range, when measuring with conventional techniques, and only a fraction of the possible Reynolds number range in the Superpipe can be investigated.

The only solution, in order to avoid spatial filtering (except building a larger facility), is to make the sensors smaller. This was done by Bailey et al. (2010) and later substantially improved by Vallikivi et al. (2011), who manufactured sensors more than one order of magnitude smaller than conventional hot wires. These new sensors were deployed by Hultmark et al. (2012) to investigate the streamwise fluctuations over an unprecedented Reynolds number range $\left(2 \times 10^{3}<R^{+}<1 \times 10^{5}\right)$, with minimal spatial-filtering effects. Several interesting features can be observed in that dataset, but perhaps the most interesting was the correspondence between the logarithmic regions between the fluctuations and the mean velocity, which indicated that the scaling between the mean flow and the fluctuations is much more similar than previously believed. Figure 1 shows that both the variance and the mean velocity 


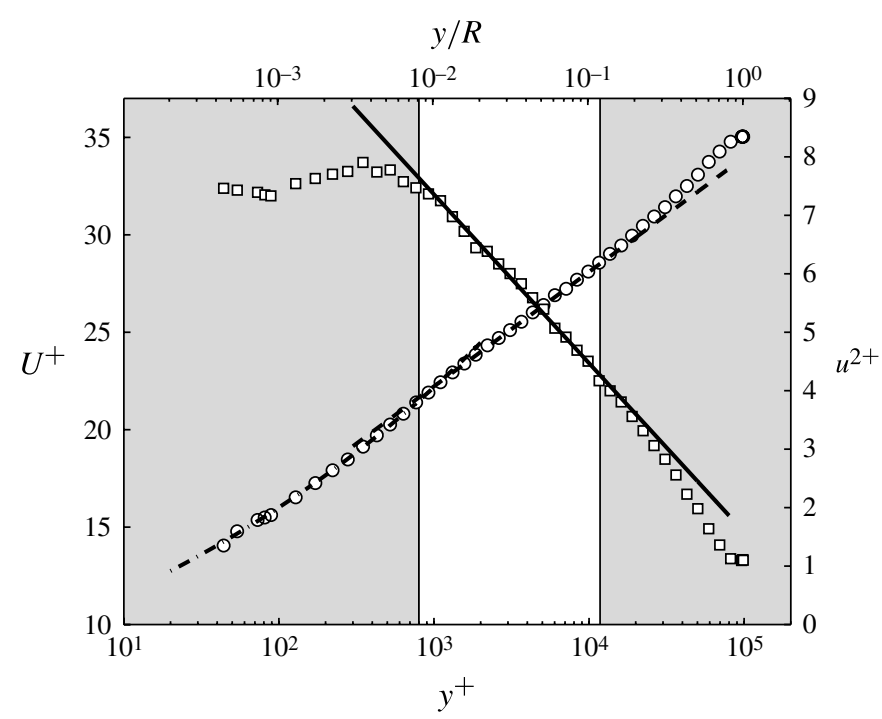

FIGURE 1. Comparison of mean velocity and turbulence streamwise fluctuation, where a clear similarity can be seen. The white area indicates the identified logarithmic region. $\circ$, mean velocities; $\square$, turbulence fluctuations. Dashed line indicates the log law for the mean velocity and the dash-dotted line is the power law for the mean velocities as described by McKeon et al. (2004). The solid line is the log law for the fluctuations as proposed by Hultmark et al. (2012). Figure adopted from Hultmark et al. (2012).

exhibit a logarithmic profile in the same region in space and that the layers identified in the mean velocity can also be identified in the variance profile. The logarithmic behaviour of the variances was predicted by Townsend (1976), who used his attachededdy hypothesis combined with dimensional analysis to arrive at the scaling law. Later Perry, Henbest \& Chong (1986) derived a similar logarithmic behaviour using an overlap argument on the wavenumber spectrum, which yields the variance if integrated over all wavenumbers. The logarithmic law for the variances takes the form:

$$
\frac{\overline{u^{\prime 2}}}{\overline{u_{\tau}^{2}}}=B_{1}-A_{1} \log (\bar{y}) \text {. }
$$

Hultmark et al. (2012) found the constants, by a regression fit, to be $A_{1}=1.25$ and $B_{1}=1.61$. However, in contrast to the logarithmic law for the mean velocities, where the coefficient, $\kappa$, can be related to the friction factor (Zagarola \& Smits 1998; Wosnik et al. 2000), the derivations by Townsend and Perry et al. give no insight into the actual value of the coefficient $A_{1}$. In this paper a theory for the scaling of the streamwise turbulence fluctuations - similar to that proposed by Wosnik et al. for the mean velocities - will be developed, with a resulting formulation of the constant $A_{1}$. The proposed theory is strongly supported by the unique high Reynolds number dataset by Hultmark et al. (2012).

\section{Theory}

Despite the striking similarity between the velocity fluctuations and the mean velocity as shown by Hultmark et al. (2012), the classical derivation for the mean velocity, as outlined in $\S 1$, is not applicable to the turbulent fluctuations. The 
reason is that the Reynolds number dependent offset, $U_{c l} / u_{\tau}$, introduced when nondimensionalizing the mean velocity in the outer region has no equivalence in the case of the fluctuations. Since no obvious offset can be subtracted from the fluctuations, they will be a strong function of Reynolds number, which can be seen as the offset indicated by an arrow in figure 2. In order to account for this Reynolds number shift, one can allow the non-dimensionalized variances to be functions of Reynolds number, $R^{+}$, similar to the strategy of Wosnik et al. (2000) in their derivation of the mean velocity scaling:

$$
\frac{\overline{u^{\prime 2}}}{u_{\tau}^{2}}=g_{i}\left(y^{+}, R^{+}\right)
$$

and

$$
\frac{\overline{u^{\prime 2}}}{u_{\tau}^{2}}=g_{o}\left(\bar{y}, R^{+}\right)
$$

It is important to point out that these two functions describe the profile throughout the shear layer, not only in the inner or outer regions, as is the case in the traditional argument. By introducing an intermediate variable $\tilde{y}=y^{+}{R^{+}}^{-n}$ and combining (2.1) and (2.2), we obtain

$$
g_{o}\left(R^{+n-1} \tilde{y}, R^{+}\right)=g_{i}\left(R^{+n} \tilde{y}, R^{+}\right)
$$

For $0<n<1$, it is possible, by fixing $\tilde{y}$, to remain physically located in the overlap region in the limit as $R^{+} \rightarrow \infty$ while simultaneously letting $y^{+} \rightarrow \infty$ and $\bar{y} \rightarrow 0$. By differentiating (2.3) with respect to $R^{+}$(while keeping $\tilde{y}$ constant), following the procedure in Wosnik et al., one can show that

$$
\left.\bar{y} \frac{\partial g_{o}}{\partial \bar{y}}\right|_{R^{+}}=-\left[\left.\frac{\partial g_{i}\left(y^{+}, R^{+}\right)}{\partial \log R^{+}}\right|_{y^{+}}-\left.\frac{\partial g_{o}\left(\bar{y}, R^{+}\right)}{\partial \log R^{+}}\right|_{\bar{y}}\right]=-S_{f} .
$$

The term in square brackets on the right-hand side has the same form as the sensitivity function for the mean velocity, as introduced by Wosnik et al. (2000), but here for the fluctuations (referred to as the sensitivity function for the fluctuations, $S_{f}$, from here on). If the above equation is integrated with respect to $\bar{y}$, the result will be a logarithmic profile, as predicted by Townsend (1976) and Perry et al. (1986) and experimentally observed by Hultmark et al. (2012), only if the sensitivity function is constant over a region in space. Additionally, the magnitude of the coefficient of the logarithmic law $\left(A_{1}\right.$ in (1.9)) will take the value of the magnitude of the sensitivity function, such that $A_{1}=S_{f}$, in the region where $S_{f}$ is constant. However, by inspecting figures 2 and 3 one can conclude that the main contribution to the total magnitude of the sensitivity function, $S_{f}$, will come from the inner-scaled function, $g_{i}$, since the outer-scaled function, $g_{o}$ is fairly well collapsed, at least for the higher Reynolds numbers.

Since (2.4) must be independent of origin in $y$ the most general form of the logarithmic behaviour is

$$
\frac{\overline{u^{\prime 2}}}{u_{\tau}^{2}}=B_{0}-S_{f} \log \left(y^{+}+b^{+}\right)
$$




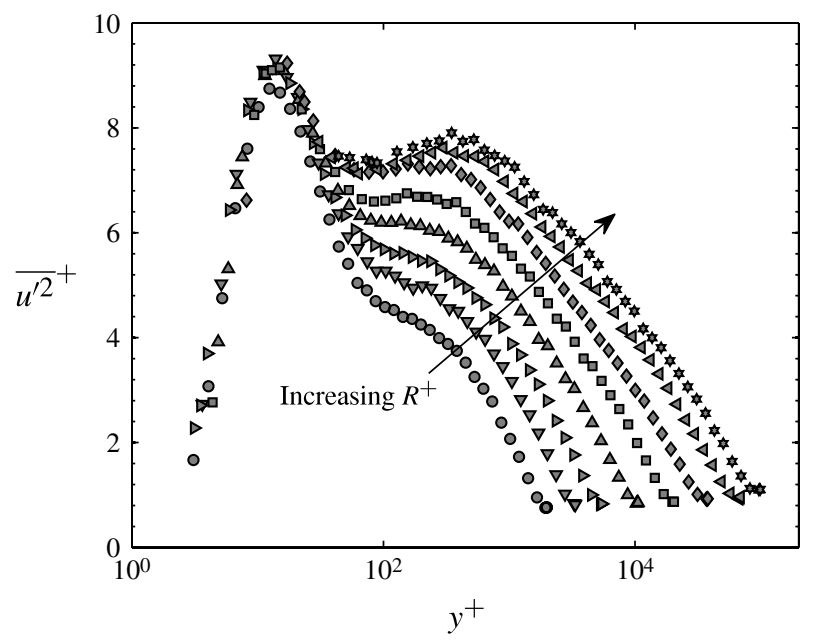

FIGURE 2. Inner-scaled turbulence fluctuations for $R^{+}=1985,3334,5411,10481,20251$, 37 450, 68371 and 98 187. Arrow indicates the Reynolds number offset in the overlap region. Data from Hultmark et al. (2012).

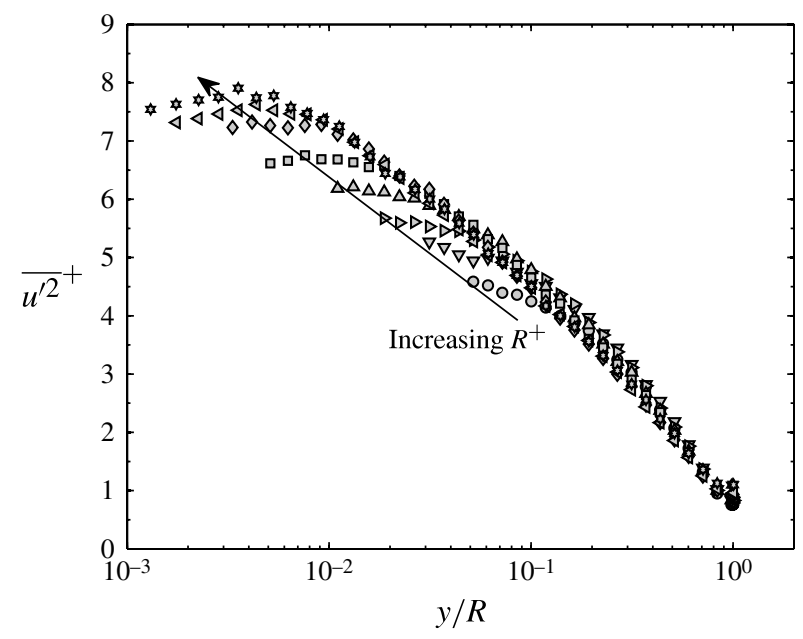

FIGURE 3. Outer-scaled turbulence fluctuations for $R^{+}=1985,3334,5411,10481,20251$, 37450,68371 and 98187 . It is clear that the collapse in the overlap region is better for the outer scaling than inner scaling. Data from Hultmark et al. (2012).

and similarly

$$
\frac{\overline{u^{\prime 2}}}{u_{\tau}^{2}}=B_{1}-S_{f} \log (\bar{y}+\bar{b}),
$$

where $B_{0}, B_{1}, \bar{b}$ and $b^{+}$can, at most, be functions of Reynolds number; $b^{+}$and $\bar{b}$ are equivalent to the offsets introduced in the mean velocity which describe the mesolayer. From a mathematical perspective the offset for the fluctuations does not need to be identical to that of the mean flow. However, since they are both signs of viscous 
$\bar{y}$

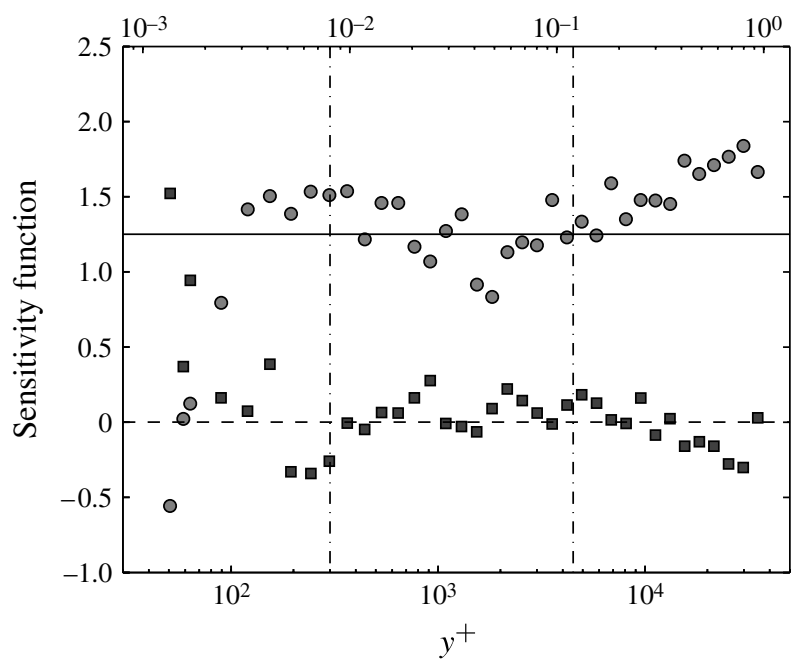

FIGURE 4. The sensitivity functions for the mean velocity, $S_{m}(\boldsymbol{\square})$, and the fluctuations, $S_{f}$ $(\bullet)$, calculated for $R^{+}=68371$. The solid line indicates the value of $A_{1}$ as found by regression fit in Hultmark et al. (2012) and the dashed line marks the zero level. The dash-dotted lines indicate the flat region $\left(300<y^{+}<0.12 R^{+}\right)$.

effects on the profiles one would expect them to be of the same order of magnitude. This theory does not reveal the value of the two additive constants $B_{0}$ and $B_{1}$ but they can be related to each other as (by using the fact that $y^{+}=\bar{y} R^{+}$)

$$
B_{0}=B_{1}+S_{f} \log R^{+}
$$

\section{Validation and discussion}

First, the sensitivity functions will be studied, since both the theory by Wosnik et al. and the theory proposed above indicate that they play a very important role in the formation of the logarithmic layers, both in the mean velocity and the fluctuations. Both $S_{m}$ and $S_{f}$ have to be nearly constant over a region in space for the logarithmic behaviour to appear (in the region where the respective sensitivity functions are constant).

Figure 4 shows the two sensitivity functions, $S_{m}$ and $S_{f}$, as a function of $y^{+}$for the second highest Reynolds number tested in Hultmark et al. (2012) $\left(R^{+}=68371\right)$. The derivatives are estimated by a double-sided finite-difference scheme where the data at $R^{+}=37450$ and $R^{+}=98187$ have been linearly interpolated to match the $y^{+}$ and $\bar{y}$ positions of the $R^{+}=68371$ case. One can see that both $S_{m}$ and $S_{f}$ exhibit an approximately constant region between $300<y^{+}<8000$ (which corresponds to $0.003<\bar{y}<0.12$ ). In this region their magnitudes are $S_{m}=0.01 \pm 0.3$ and $S_{f}=1.27 \pm 0.4$. Thus, to within experimental error, the mean velocity can be expected to have logarithmic behaviour with a slope of $1 / \kappa$ and the turbulence fluctuations can be expected to have a logarithmic behaviour with a slope of $A_{1}=1.27 \pm 0.4$. This is in close agreement with results by Hultmark et al. (2012), who found $A_{1}=1.25$ by a regression fit of the experimental data. 

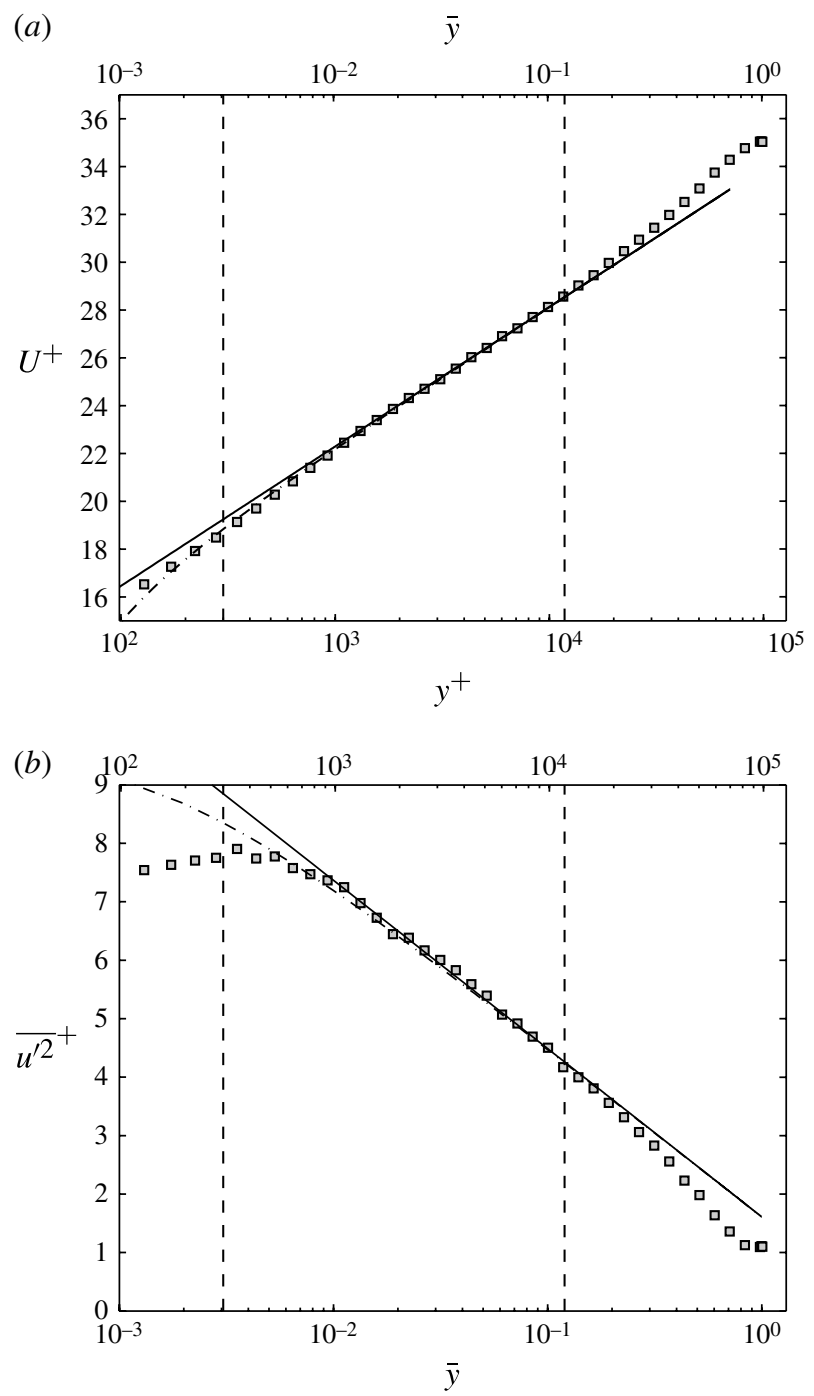

FIGURE 5. Profiles of $(a)$ mean velocity and $(b)$ streamwise turbulence fluctuations for $R^{+}=$ 98 187. Solid lines indicate a logarithmic behaviour, dash-dotted lines indicate logarithm with offset $\left(a^{+}=45\right.$ and $\left.\bar{b}=0.0015\right)$. Dashed lines indicate the range for which the sensitivity function is approximately constant $\left(300<y^{+}<0.12 R^{+}\right)$.

The outer limit of the flat region of the sensitivity functions $(\bar{y} \approx 0.12)$ is in near perfect agreement with the outer limit found for the two logarithmic regions. However, the inner limit is lower for the sensitivity functions $\left(y^{+}>300\right)$ than for the logarithmic region observed in the experimental data, which was found for $y^{+}>800$. Thus, the overlap region extends further than the inertial region, which can be explained by the introduction of the offset in the logarithmic functions (as shown in (1.6) and (2.6), for the mean velocity and turbulence fluctuations respectively). The extents of the regions with constant $S_{m}$ and $S_{f}$ were selected manually and are subject to large uncertainties since the deviation from a constant value is subtle (especially at the upper limit). Figure 5(a) shows the mean velocity profile plotted as function of $y^{+}$, 
compared to classical logarithmic behaviour and that including an offset $a^{+}=-45$, thus allowing for a mesolayer. It should be pointed out that the value of $a^{+}$found here is larger than that found by Wosnik et al. (2000), which is in agreement with the observation that the logarithmic region begins further away from the wall as noted by Hultmark et al. (2012). It is clear that including the offset extends the logarithmic behaviour throughout the whole region for which $S_{m} \approx 0$. Similarly for the turbulence fluctuations, figure $5(b)$ shows the log law with and without an offset $(\bar{b}=0.0015)$. The introduction of an offset increases the range of collapse and the inner limit is close to that for the sensitivity functions. Thus, a mesolayer exists for approximately $300<y^{+}<800$, where the turbulence is still affected by viscosity, and between $800<y^{+}<0.12 R^{+}$a true inertial region exists where both profiles exhibit logarithmic behaviours.

The theory proposed above extends the similarities between the mean and the fluctuations as seen in experimental data to also include the theoretical derivation, where both $\log$ laws can be derived in a similar manner. Additionally, it allows a description of the slope of the log law for the fluctuations, which agrees very well with the experimental data.

\section{Acknowledgements}

The author would like to thank W. K. George for all the useful hints and discussions and A. J. Smits for continuing use of the Superpipe data. This paper was written while the author was working with M. B. Parlange in the Laboratory of Environmental Fluid Mechanics and Hydrology at École Polytechnique Fédérale de Lausanne (EPFL).

\section{REFERENCES}

Bailey, S. C. C, Kunkel, G. J., Hultmark, M., Vallikivi, M., Hill, J. P., Meyer, K. A., Tsay, C., Arnold, C. B. \& Smits, A. J. 2010 Turbulence measurements using a nanoscale thermal anemometry probe. J. Fluid Mech. 663, 160-179.

CAstillo, L. 1997 Similarity analysis of turbulent boundary layers. PhD thesis, State University of New York at Buffalo.

Frisch, U. \& Orszag, S. A. 1990 Turbulence: challenges for theory and experiment. Phys. Today 43 (1), 24-32.

George, W. K. 1988 Another look at the log (or is it a power law?) velocity profile for a zero-pressure gradient boundary layer.. Bull. Am. Phys. Soc. 33 (10), 2301.

George, W. K. 2007 Is there a universal log law for turbulent wall-bounded flows?. Phil. Trans. R. Soc. Lond. A 365, 789-806.

George, W. K. \& CAStillo, L. 1997 Zero-pressure-gradient turbulent boundary layer. Appl. Mech. Rev. 50, 689-729.

Hultmark, M. N., Bailey, S. C. C. \& Smits, A. J. 2010 Scaling of near-wall turbulence in pipe flow. J. Fluid Mech. 649, 103-113.

Hultmark, M., Vallikivi, M., Bailey, S. C. C. \& Smits, A. J. 2012 Turbulent pipe flow at extreme Reynolds numbers. Phys. Rev. Lett. 108, 094501.

Hutchins, N., Nickels, T. B., Marusic, I. \& Chong, M. S. 2009 Hot-wire spatial resolution issues in wall-bounded turbulence. J. Fluid Mech. 635, 103-136.

McKeon, B. J., Li, J., Jiang, W., Morrison, J. F. \& Smits, A. J. 2004 Further observations on the mean velocity distribution in fully developed pipe flow. J. Fluid Mech. 501, 135-147.

MillikAN, C. B. 1939 A critical discussion of turbulent flows in channels and circular tubes. In Proceedings of the Fifth International Congress of Applied Mechanics. Cambridge, MA.

Morrison, J. F., McKeon, B. J., JiAng, W. \& Smits, A. J. 2004 Scaling of the streamwise velocity component in turbulent pipe flow. J. Fluid Mech. 508, 99-131. 
Perry, A. E., Henbest, S. M. \& Chong, M. S. 1986 A theoretical and experimental study of wall turbulence. J. Fluid Mech. 165, 163-199.

Smits, A. J. (ED.) 1991 Proceedings of the Princeton Workshop on New Approaches to Experimental Turbulence Research. Princeton, NJ.

Smits, A. J., Monty, J., Hultmark, M., Bailey, S. C. C., Hutchins, M. \& Marusic, i. 2011 Spatial resolution correction for turbulence measurements. J. Fluid Mech. 676, 41-53.

SQuire, H. B. 1948 I. reconsideration of the theory of free turbulence. Phil. Mag. (7) 39 (288), $1-20$.

Stanton, T. E. \& Pannell, F. R. S. 1914 Similarity of motion in relation to the surface friction of fluids. Phil. Trans. R. Soc. Lond. A 214, 199-212.

Townsend, A. A. 1976 The Structure of Turbulent Shear Flow. Cambridge University Press.

Vallikivi, M., Hultmark, M., Bailey, S. C. C. \& Smits, A. J. 2011 Turbulence measurements in pipe flow using a nano-scale thermal anemometry probe. Exp. Fluids 51, 1521-1527.

WosNiK, M. 2000 On wall-bounded turbulent shear flows. PhD thesis, State University of New York at Buffalo.

Wosnik, M., Castillo, L. \& George, W. K. 2000 A theory for turbulent pipe and channel flows. J. Fluid Mech. 421, 115-145.

Zagarola, M. V. 1996 Mean-flow scaling of turbulent pipe flow. PhD thesis, Princeton University.

Zagarola, M. V. \& SMIts, A. J. 1997 Log laws or power laws: the scaling in the overlap region. Phys. Fluids 9, 2094.

Zagarola, M. V. \& SMits, A. J. 1998 Mean-flow scaling of turbulent pipe flow. J. Fluid Mech. 373, 33-79. 